\title{
EFFECT OF STRUCTURAL RELAXATIONS WITHIN THE AMORPHOUS STRUCTURE ON THE MAGNETIC PROPERTIES OF AMORPHOUS TAPES FROM FECOB FAMILY
}

\begin{abstract}
The paper presents the research results for the $\mathrm{Fe}_{78} \mathrm{Co}_{2} \mathrm{Si}_{9} \mathrm{~B}_{11}$ amorphous alloy, and after the process of annealing resulting only in the relaxation of the material. The structure relaxations occurring in the volume of test samples lead to the changes in their magnetic and mechanic properties. Therefore the studies on the effect of the structure defects on the properties of these type of materials are important. Understanding the processes occurring during the magnetizing of amorphous alloys can be helpful in the design of modern functional materials for special purposes. The main purpose of this elaboration was to determine the effect of the amorphous structure defects in the state after solidification and after heat treatment on the changes in the magnetizing process and in such parameters as the saturation magnetization and the coercivity field.

Keywords: amorphous materials, free volumes, quasidislocation dipoles, scanning electron microscopy, approach to ferromagnetic saturation, magnetic moments
\end{abstract}

\section{Introduction}

The molecular structure of amorphous alloys is completely different from the one observed in the metal components found in our surroundings. This very diversity in the arrangement of atoms in the volume of amorphous alloys causes their exceptional properties [1-5]. In contrast to the crystalline structure, where interactions between atoms have a long-range character, and their arrangement is symmetrical and maintains the angle translation with periodicity, in amorphous materials atoms are arranged chaotically and are subject to interactions of short or medium range [6]. Studies on the amorphous alloys have been conducted for over 50 years [7-8]. Initially thin metallic layers were manufactured, and over time a production technique was developed, which allowed to obtain amorphous samples in the form of thin tapes of thickness not exceeding $100 \mu \mathrm{m}$ [9-10]. Those samples were interesting enough to become a research material for materials engineering and physics specialists. FeCoB based amorphous alloys that demonstrate so called soft magnetic properties proved to be of special interest [11-14]. The amorphous tapes based on $\mathrm{FeCoB}$ experience significantly lower losses during magnetization reversal than FeSi transformer steels used in the electro technical industry [15]. In addition these materials demonstrate almost zero magnetostriction and, depending on the chemical composition, relatively high saturation magnetization. Another group of materials which can be successfully used in electrotechnics are the nanocrystalline alloys, manufactured from amorphous alloys by a proper heat treatment [16-20]. However, it is not always the case, that introducing tiny crystalline grains of nanometric size into amorphous solid positively affects its magnetic properties, and in particular magnetostriction phenomenon. Therefore, soft magnetic properties of amorphous alloys can be enhanced by using a proper heat treatment, which does not result in their nanocrystallization but only the relaxation of the structure, or by extending the time of their solidification [21-23]. The temperatures for the heat treatment are usually specified by the analysis of results of heat flow in the function of temperature (calorimetric-DSC) and the time is determined experimentally [24]. There are several types of heat treatment: impulse, short-term, and long-term [25-28]. It is difficult to describe them accurately, as the important parameter of those processes of annealing, besides the time, is also the temperature, which can take different values, depending on the demanded process. Therefore the heat treatment can be classified as hightemperature and low-temperature.

This paper presents the results of studies on amorphous samples of $\mathrm{Fe}_{78} \mathrm{Co}_{2} \mathrm{Si}_{9} \mathrm{~B}_{11}$ alloys in the form of tapes, in the state both after solidification and after the heat treatment in the resistance furnace under vacuum. The paper includes the research results for the structure and magnetic properties and lists their explanation in relation to the relaxation processes occurred in their volume. The assumptions of the approach to ferromagnetic saturation theory developed by H. Kronmüller was used. 


\section{Research procedure}

The research samples were produced using the method of unidirectional cooling of the liquid alloy on the copper rotating wheel. The production process was carried out in protective atmosphere (Ar). The alloy components were of high purity: $\mathrm{Fe}$ $-99.98 \%$ atm., Co - $99.98 \%$ atm., $\mathrm{Si}-99.98 \%$ atm. Boron was added in the form of $\mathrm{Fe}_{45.4} \mathrm{~B}_{54.6}$ alloy in order to avoid processing problems with boron melting. The temperatures of the heat treatment were determined on the basis of the analysis of heat flow curves in the function of temperature (DSC). Selected temperature was $610 \mathrm{~K}$ and the annealing time $15 \mathrm{~min}$. The differential scanning calorimeter (NETZSCH), used in the research, allowed the measurement up to the liquidus temperature for the samples tested. The annealing of samples was performed in a vacuum furnace providing appropriate conditions them prevented the oxidation of samples. The structure of alloys was tested using the X-ray diffraction and the scanning electron microscopy. The samples were exposed to X-rays for $5 \mathrm{~s}$ for one measurement step equal to $0,02^{\circ}$ in $2 \Theta$ angle range of $30^{\circ}$ to $100^{\circ}$. X-ray measurements were performed using BRUKER ADVANCE D8 machine equipped with a lamp with copper cathode (CuKõ 1.54178). The scanning electron microscopy was carried out using Supra 35 microscope manufactured by Zeiss. The images were obtained using the detection of secondary electrons (SE) of accelerating voltage of $25 \mathrm{kV}$ and maximum magnification of $x 25000$. The initial magnetization curves were measured using a vibrating magnetometer (VSM - LAKESCHORE 7301 ), working in the magnetic field with the maximum strength of $2 \mathrm{~T}$.

\section{Theoretical introduction}

Both in amorphous and crystalline materials we observe structure defects which affect their properties [28-29]. While in the case of crystalline materials such defects are well defined based on group models, there arises a difficulty to identify them precisely in amorphous materials. The reason is it is difficult to refer to a molecular structure that is chaotic, ie. there is no order in the arrangement of atoms with repeatability and angular correlations. The lack of a pattern, ie. the reference point is a major barrier for the evaluation of the amorphous structure. There are several possible methods for such research, but they are time consuming and very expensive (long-term X-ray measurements with the use of unpopular lamps with molybdenum or silver cathodes or synchrotron measurements). In the case of amorphous materials being ferromagnetic materials with soft magnetic properties, their structure can be tested using an indirect method consisting in analyzing the initial magnetization curves. This analysis is performed according to the assumptions of H. Kronmüller theory, developed on the basis of W.H. Brown elaboration for microcrystalline model [30-31]. H. Kronmüller assumed that in amorphous alloys there occur local differences of the composition and density [32-33]. In those areas there occurred more or less magnetic atoms, which after upon application of magnetic field of sufficiently high strength underwent the magnetization vector rotation, in accordance with the magnetic field direction. Unfortunately the barrier for their fast redirection are the very structure defects. In the volume of amorphous alloys there can be two types of structure defects: free volumes (Fig. 1) and quasidislocation dipoles (Fig. 2) [34-36]. Free volumes and quasidislocation dipoles in amorphous alloys can be treated like point defects and linear defects in crystalline materials, respectively. a)

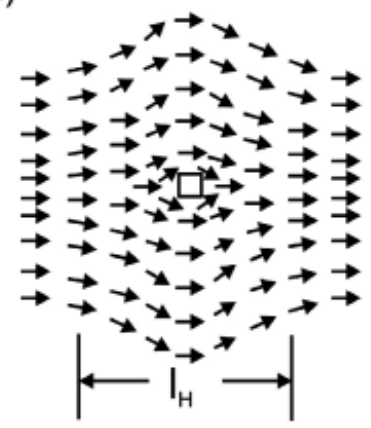

b)

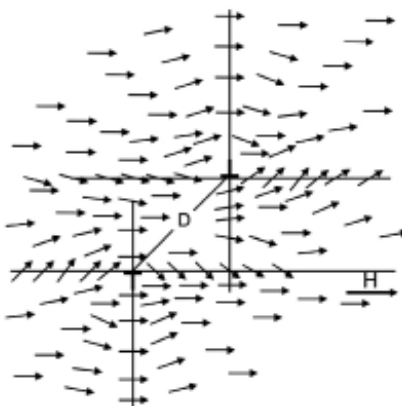

Fig. 1. Diagram illustrating the distribution of magnetic moments around the free volume [34-36] a)

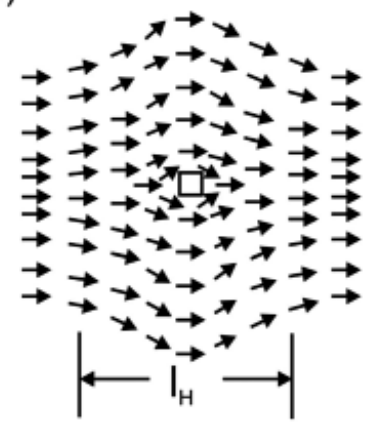

b)

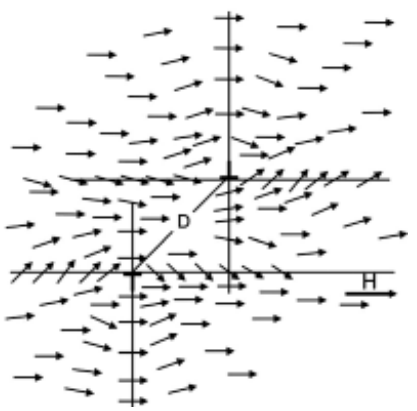

Fig. 2. Diagram illustrating the distribution of magnetic vectors within the range of quasidislocation dipole [34-36]

In magnetic fields above the effective anisotropy the magnetizations can be described with the formula (1) [37]:

$$
\begin{aligned}
M(H)= & M_{s}\left[1-\frac{a_{1 / 2}}{\left(\mu_{0} H\right)^{1 / 2}}-\frac{a_{1}}{\left(\mu_{0} H\right)^{1}}-\frac{a_{2}}{\left(\mu_{0} H\right)^{2}}\right]+ \\
& +b\left(\mu_{0} H\right)^{1 / 2}
\end{aligned}
$$

where: $M_{S}-$ the saturation magnetization, $\mu_{0}$ - the permeability constant, $H$ - the strength of magnetic field, $a_{i}(I=1 / 2,1,2)$ - coefficients of the linear fit, correspond to free volumes and linear defects, the b-coefficients of the linear fit corresponding to the suppression of spin waves by a magnetic field of high strength - the Holstein-Primakoff paraprocess [38]. 


\section{Research results and discussion}

Fig. 3 presents the images for X-ray diffraction on the preparation made for the $\mathrm{Fe}_{78} \mathrm{Co}_{2} \mathrm{Si}_{9} \mathrm{~B}_{11}$ alloy samples tested.

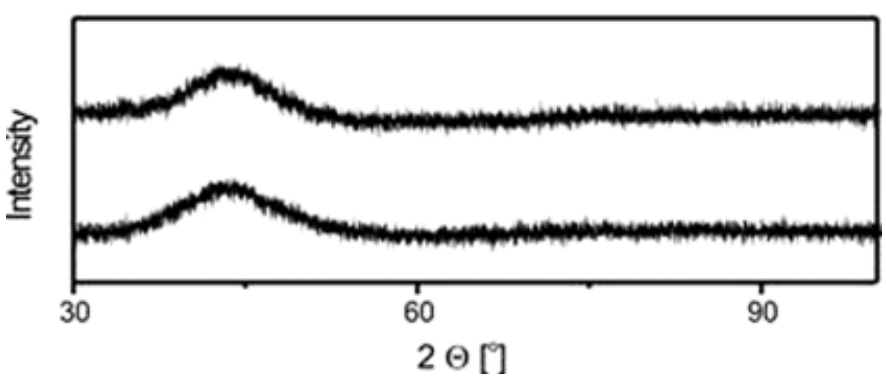

Fig. 3. X-ray diffractograms obtained for the samples of $\mathrm{Fe}_{78} \mathrm{Co}_{2} \mathrm{Si}_{9} \mathrm{~B}_{11}$ alloy samples tested: a) in the state after solidification, b) after heat treatment $610 \mathrm{~K} / 15 \mathrm{mins}$

As can be seen in Fig. 3, X-ray diffraction images for the sample in the state after solidification and after heat treatment are similar. They consist only of a single wide maximum, called the amorphous halo. Such a shape of diffractograms is typical for materials with the amorphous structure.

In addition the structure of the alloys was observed using scanning electron microscopy (Fig. 4).
The fractures of tapes presented in Fig. $4 \mathrm{a}$ and $4 \mathrm{~b}$ are slightly different and demonstrate the contribution of performed heat treatment. The magnifications of selected fragments of the fractures (Fig. 4c and 4d) reveal the structures of changing character, proving different plasticity of tested tapes in places shown. The nature of amorphous alloys relates to the chemical and typological disorder and to close-range interactions between atoms. Therefore, there are locally observed areas of changing chemical composition, confirming the existence of composition and density fluctuations in the volume of amorphous alloys. In Fig. $4 \mathrm{c}$ there are three basic structures describing fractures for amorphous alloys that can be distinguished. In the upper part of the fracture, starting around the middle of the tape there is a dominant plain fracture with slightly uneven surface. In the bottom part of Fig. $4 \mathrm{c}$ there can be observed a smooth transition with visible plate faults of the surface. In the case of the sample after isothermal annealing the fracture does not contain the areas of plain structure. This fracture has an extensive surface across the whole area with one vertical fault. Such a fracture nature is typical for a ductile fracture with the occurrence of tiny veins and small scales.

The magnetic properties of the alloys, such as the saturation magnetization and the value of coercivity field were determined based on the analysis of static loops of magnetic hysteresis (Fig. 5).

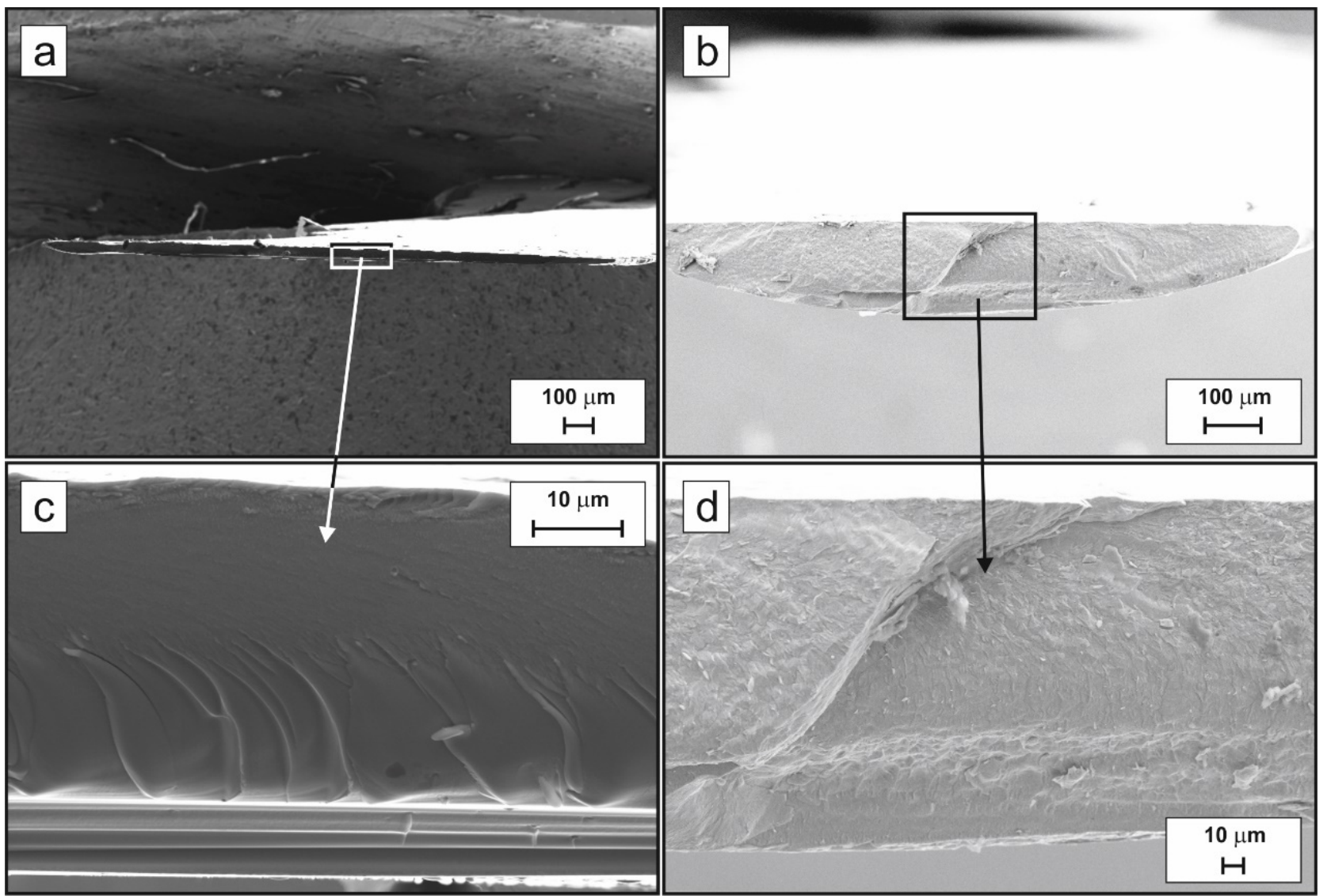

Fig. 4. Images illustrating fractures of tested tapes of $\mathrm{Fe}_{78} \mathrm{Co}_{2} \mathrm{Si}_{9} \mathrm{~B}_{11}$ alloy: a, c) in the state after solidification, b, d) after heat treatment $610 \mathrm{~K} / 15$ mins 


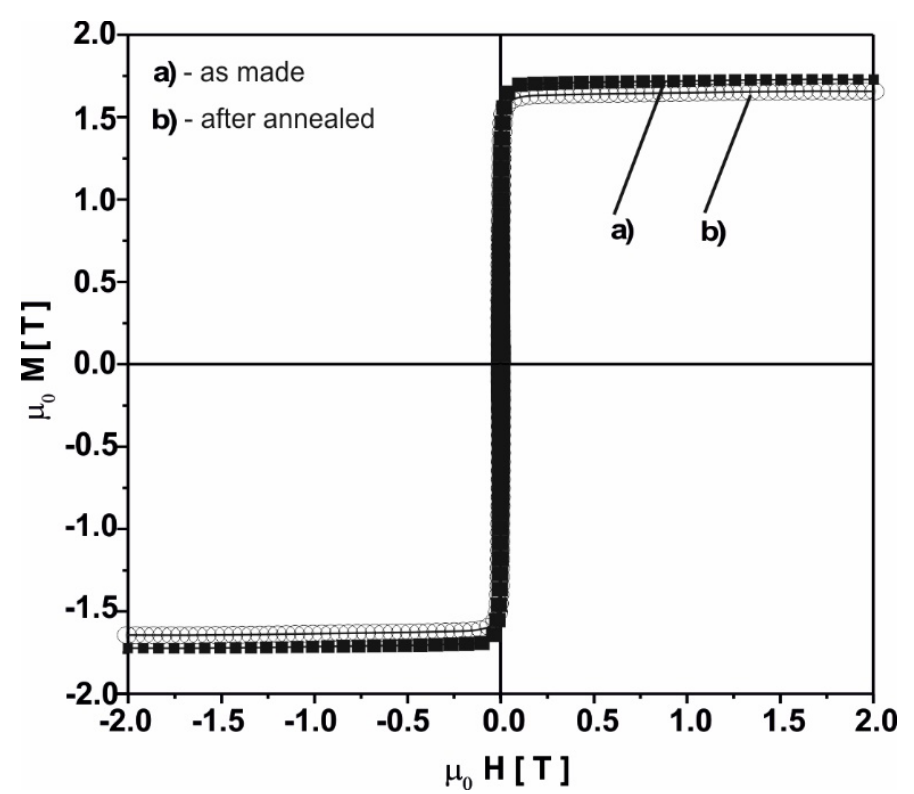

Fig. 5. Static loops of magnetic hysteresis measured for $\mathrm{Fe}_{78} \mathrm{Co}_{2} \mathrm{Si}_{9} \mathrm{~B}_{11}$ alloy: a) in the state after solidification, b) after heat treatment $610 \mathrm{~K} / 15 \mathrm{mins}$

Magnetic hysteresis loops shown in Fig. 5 are typical for ferromagnetic materials demonstrating soft magnetic properties. Tested material before undergoing isothermal annealing $610 \mathrm{~K} / 15 \mathrm{mins}$ had a high saturation magnetization equal as much as $1,63 \mathrm{~T}$. After the relaxation of the structure consisting in releasing relaxators into the surface of the sample and their conglomeration in the volume of the alloy, there was a slight change in the saturation magnetization value of $1.69 \mathrm{~T}$ observed. The annealing in the temperature well below the crystallization temperature had quite a large effect on the value of coercivity field. Events having a particular effect on so called magnetic softness (the width of static loop of magnetic hysteresis in the middle part of M-H system) can be tested in the area above the effective anisotropy field, called the approach to ferromagnetic saturation. Data obtained from the analysis of static loops of magnetic hysteresis was collected in Table 1.

TABLE 1

The saturation magnetization $-M_{s}$ and the value of coercivity field $-H_{c}$ determined from the analysis of static loops of magnetic hysteresis

\begin{tabular}{|c|c|c|}
\hline Pamples & $\begin{array}{c}\boldsymbol{M}_{\boldsymbol{s}} \\
{[\mathbf{T}]}\end{array}$ & $\begin{array}{c}\boldsymbol{H}_{\boldsymbol{c}} \\
{[\mathbf{A} / \mathbf{m}]}\end{array}$ \\
\hline As cast state & 1.63 & 474 \\
\hline After annealed & 1.69 & 118 \\
\hline
\end{tabular}

The value of coercivity field changes as a result of blocking the free movement of domain walls in the volume of amorphous alloys. Observed changes are the consequence of composition and density fluctuation described by topological and chemical disorder for close-range interactions between atoms. In the case of isothermal annealing, resulting only in the relaxation of the structure, there occurs the decrease of free system energy. If the selected heat treatment time and temperature result in the diffusion of atoms to greater distances, the volume of the alloy may be subject to the creation of areas, where the interactions between atoms are of medium-range nature. Such systems emerge locally in preferential places, where the provision of sufficiently large energy results in building up additional atoms in clusters, while medium-range interactions are replaced by far-range interactions. Those areas constitute a crystalline seed, which later converts into crystalline grain.

The change in saturation magnetization is directly related to the annealing process leading to the rearrangement of magnetic atoms in the volume of tapes tested. The use of results of initial magnetization curves analysis allows to obtain direct information on the parameter describing the stiffness of spin wave $\left(D_{\mathrm{spf}}\right)$, which is related to the amount of magnetic moments in given volume. For the test samples in the state after solidification and the process of isothermal annealing, the process of magnetizing was observed primarily in the range of point defects. The amount of these defects in relation to the sample in the state after solidification decreased slightly, which means that the dominant principle of the approach to ferromagnetic saturation in the time of magnetizing was the first principle defined with the relation $\left.\mu_{0} \mathrm{Ms}-\mu_{0} \mathrm{H}\right)^{1 / 2}$. In higher magnetic fields, where the magnetizing process is no longer related to the non-collinear movement of magnetization vectors around the structure defects called free volumes, the leading role is taken by the phenomenon consisting in the suppression of thermally induced spin waves, ie. the socalled Holstein-Primakoff paraprocess [38]. Fig. 6 presents the linear relation fits describing the first principle of the approach to ferromagnetic saturation for test alloy samples in the state after solidification and after heat treatment.

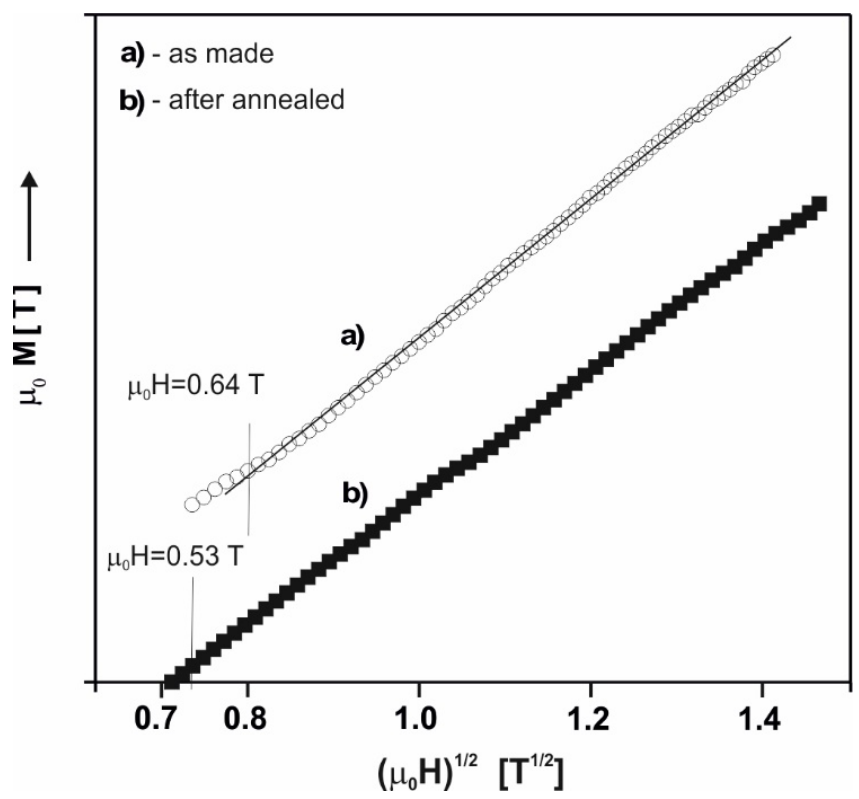

Fig. 6. Relations describing the Holstein-Primakoff paraprocess: a) in the state after solidification, b) after heat treatment $610 \mathrm{~K} / 15 \mathrm{mins}$

The value of the stiffness of spin wave parameter was determined based on the calculations carried out in accordance 
to $[32,39-40]$. After the completion of the annealing process resulting in the relaxation of the material the value of the stiffness of spin wave parameter increased, which is consistent with previous assumptions. The data obtained from the analysis of the initial magnetization curve according to the principle describing the quasi-effect was collected in Table 2.

TABLE 2

Data obtained from the analysis of reduced magnetization as a function of magnetic field strength in powers of: $1 / 2$. The parameter $b$ are the fit parameters of reduced magnetization as functions of $\left(\mu_{0} \mathrm{H}\right) 1 / 2$, Dspf - stiffness of spin wave parameter

\begin{tabular}{|c|c|c|}
\hline \hline \multicolumn{3}{|c|}{$\mathbf{F e}_{\mathbf{7 8}} \mathbf{C o}_{\mathbf{2}} \mathbf{S i}_{\mathbf{9}} \mathbf{B}_{\mathbf{1 1}}$} \\
\hline State of sample & $\begin{array}{c}\mathbf{b} \\
{\left[\mathbf{T}^{\mathbf{1} 2}\right]}\end{array}$ & $\begin{array}{c}\mathbf{D}_{\text {spf }} \\
{\left[\mathbf{1 0}^{-2} \mathbf{m e V n m}{ }^{2}\right]}\end{array}$ \\
\hline As made & 0.029 & 71 \\
\hline Annealed & 0.033 & 64 \\
\hline
\end{tabular}

\section{Conclusions}

The samples manufactured using the technique of unidirectional cooling of the liquid alloy on the copper rotating wheel, in the form of tapes, demonstrated the amorphous structure. The annealing of samples did not result in their crystallization, but only to the relaxation of the structure. The level of structure relaxation and changes in the plasticity and in particular in the ductility of alloys can be deducted indirectly from the evaluation of the structure of fractures. There are four characteristic fracture types in amorphous alloys: plain, vein, scale and river fractures. The plain fracture is typical for the material of the highest level of the structure relaxation and its brittleness increase. On the other hand vein and scale fractures evidence the partial stresses in the structure and their plasticity increase. Mixed fracture can be assigned to the material of cluster build typical for amorphous materials described by topological and chemical disorder. In the test samples of $\mathrm{Fe}_{78} \mathrm{Co}_{2} \mathrm{Si}_{9} \mathrm{~B}_{11}$ alloy in the state after solidification there occurs vein fracture, rapidly transforming into the plain fracture. In the samples after annealing in the temperature of 610 $\mathrm{K}$ for 15 mins there can be observed a mixed fracture: ductile with the occurrence of tiny veins and a few scales on the entire surface of the tape fracture. Such a nature of the structure of fractures is reflected in the magnetic properties. It was observed that during the changes in the volume of amorphous alloys in the range of structure defects in the form of free volumes and quasidislocation dipoles, the movement of the magnetization vector is non-collinear. In the case of this study, both for the samples in the state after solidification and after heat treatment, the process of magnetizing in the area called the approach to ferromagnetic saturation occurred as a result of delays in magnetization vector movement caused by the presence of structure defects in the form of free volumes. The very mechanism of magnetizing was similar, and free volumes determined it in the range of magnetic field up to $0.64 \mathrm{~T}$ for the sample in the state after solidification and up to $0.53 \mathrm{~T}$ for the sample after the process of isothermal annealing $610 \mathrm{~K} / 15$ mins. Above the values of magnetic field strength describing the first principle of the approach to ferromagnetic saturation there occurred the quasi-effect. The Holstein-Primakoff paraprocess is related to the suppression of thermally induced spin waves and accompanies the further magnetization increase, up to achieving almost complete saturation magnetization.

\section{REFERENCES}

[1] Inoue, Bulk amorphous alloys: Preparation and fundamental characteristic, Mater. Sci. Fundations 6, Trans. Tech. Publications, 1-116 (1998).

[2] M. Nabiałek, Soft magnetic and microstructural investigation in Fe-based amorphous alloy, J. Alloy. Compd. 642, 98-103 (2015).

[3] M. Nabiałek, An investigation into the effect of isothermal annealing on magnetic properties in the alloy: $\mathrm{Fe}_{64} \mathrm{Co}_{10} \mathrm{Y}_{6} \mathrm{~B}_{20}$, Arch. Metall. Mater. 60(3), 1887-1891 (2015).

[4] Inoue, B.L. Shen, C.T. Chan, Fe- and Co-based bulk glassy alloys with ultrahigh strength of over $4000 \mathrm{MPa}$, Intermetallics 14, 936944 (2006).

[5] W. H. Wang, Roles of minor additions in formation and properties of bulk metallic glasses, Prog. Mater. Sci. 52, 540-596 (2007).

[6] S. Lesz, R. Babilas, M. Nabialek, M. Szota, M. Dospial, R. Nowosielski, The characterization of structure, thermal stability and magnetic properties of $\mathrm{Fe}-\mathrm{Co}-\mathrm{B}-\mathrm{Si}-\mathrm{Nb}$ bulk amorphous and nanocrystalline alloys, J. Alloy. Compd. 509, 197-201 (2011).

[7] H.S. Chen, C.E. Miller, A rapid quenching technique for the preparation of thin uniform films of amorphous solids, Rev. Sci. Instrum. 41, 1237-1238 (1970).

[8] R.B. Pond Jr., R. Maddin, A method of producting rapidly solidified filamentary castings, Trans. Metall. Soc. AIME 245, 24752476 (1969).

[9] P. Duwez and R. H. Willens, Rapid Quenching of Liquid Alloys, T. Metall. Soc. Aime 227, 362-365 (1963).

[10] T. Masumoto, R. Maddin, The mechanical properties of palladium 20 a/0 silicon alloy quenched from the liquid state, Acta Met. 19, 725-741 (1971).

[11] K. Błoch, M. Nabiałek, Approach to Ferromagnetic Saturation for the Bulk Amorphous Alloy: (Fe0.61Co0.10Zr0.025Hf0.025Ti0.02W0.02B0.20) ${ }_{97} \mathrm{Y}_{3}$, Acta. Phys. Pol. A 127 (2), 413-414 (2015).

[12] P. Pietrusiewicz, K. Błoch, M. Nabiałek, S. Walters, Influence of $1 \%$ Addition of $\mathrm{Nb}$ and $\mathrm{W}$ on the Relaxation Process in Classical Fe-Based Amorphous Alloys, Acta. Phys. Pol. A 127 (2), 397-399 (2015).

[13] M. Nabiałek, P. Pietrusiewicz, K. Błoch, Influence of the production method of $\mathrm{Fe}_{61} \mathrm{Co}_{10} \mathrm{Y}_{8} \mathrm{~W}_{1} \mathrm{~B}_{20}$ amorphous alloy on the resulting microstructure and hyperfine field distribution, J. Alloy. Compd. 628, 424-428 (2015).

[14] M. Nabiałek, P. Pietrusiewicz, M. Dośpiał, M. Szota, J. Gondro, K. Gruszka, A. Dobrzańska-Danikiewicz, S. Walters, A. Bukowska, Influence of the cooling speed on the soft magnetic and mechanical properties of $\mathrm{Fe}_{61} \mathrm{Co}_{10} \mathrm{Y}_{8} \mathrm{~W}_{1} \mathrm{~B}_{20}$ amorphous Allom, J. Alloy. Compd. 615, S56-S60 (2014). 
[15] M.E. McHenry, M.A. Willard, D.E. Laughlin, Amorphous and nanocrystalline materials for applications as soft magnets, Prog. Mater. Sci. 44, 291-433 (1999).

[16] S. Lesz, R. Szewczyk, D. Szewieczek, A. Bieńkowski, The structure and magnetoelastic properties of the Fe-based amorphous alloy with Hf addition, J. Mater. Process Tech. 157-158, 743-748 (2004).

[17] K. Błoch,M.Nabiałek, M. Dośpiał, S. Garus, Crystallization ofFe-based bulk amorphous alloys, Arch. Metall. Mater. 60(1) 7-10 (2015).

[18] D. Szewieczek, J. Tyrlik-Held, S. Lesz, Changes of mechanical properties and fracture morphology of amorphous tapes involved by heat treatment, J. Mater. Process Tech. 109, 190-195 (2001).

[19] K.M. Gruszka, M. Nabiałek, K. Błoch, J. Olszewski, Effect of heat treatment on the shape of the hyperfine field induction distributions and magnetic properties of amorphous soft magnetic $\mathrm{Fe}_{62} \mathrm{Co}_{10} \mathrm{Y}_{8} \mathrm{~B}_{20}$ alloy, Nukleonika 60(1), 23-27 (2015).

[20] D. Szewieczek, S. Lesz, Influence of structure on the evolution of magnetic and mechanical properties of amorphous and nanocrystalline $\mathrm{Fe}(85.4) \mathrm{Hf}(1.4) \mathrm{B}(13.2)$ alloy, J. Mater. Process Tech. 162, 254-259 (2005).

[21] K. Błoch, Magnetic properties of the suction-cast bulk amorphous alloy: (Fe0.61Co0.10Zr0.025Hf0.025Ti0.02W0.02B0.20) ${ }_{96} \mathrm{Y}_{4}$, J. Mang. Magn. Mater. 390, 118-122 (2015).

[22] J. Gondro, J. Swierczek, K. Błoch, J. Zbroszczyk, W.H. Ciurzynska, J. Olszewski, Microstructure and some thermomagnetic properties of amorphous and partially crystallized $\mathrm{Fe}-(\mathrm{Pt})-\mathrm{Zr}-\mathrm{Nb}$ -Cu-B alloys, Physica B: Condensed Matter 445, 37-41 (2014).

[23] J. Gondro, K. Błoch, M. Nabiałek, S. Wallters, Magnetocaloric Effect in Amorphous and Partially Crystallized Fe-Zr-Nb-Cu-B Alloy, Acta. Phys. Pol. A 127(2), 606-607 (2015).

[24] J. Gondro, K. Błoch, P. Bragiel, M. Nabiałek, M. Szota, Curie Temperature and Microstructural Changes due to the heating treatment of magnetic amorphous materials, Arch. Metall. Mater. 61, 451-456 (2016).

[25] T. Kulik, H. Matyja, Effect of flash- and furnace annealing on the magnetic and mechanical properties of metallic glasses, Mat. Sci. Eng. A, 133, 323-235 (1991).

[26] K. Błoch, M. Nabiałek, The Influence of Heat Treatment on Irreversible Structural Relaxation in Bulk Amorphous $\mathrm{Fe}_{61} \mathrm{Co}-$ ${ }_{10} \mathrm{Ti}_{3} \mathrm{Y}_{6} \mathrm{~B}_{20}$ Alloy, Acta. Phys. Pol. A 127(2), 442-444 (2015).
[27] S. Garus, M. Nabiałek, J. Garus, The Influence of Heat Treatment on Soft Magnetic Properties of the $\mathrm{Fe}_{60} \mathrm{Co}_{10} \mathrm{Mo}_{2} \mathrm{Y}_{8} \mathrm{~B}_{20}$ Alloy, Acta. Phys .Pol. A 126(4), 960-962 (2014).

[28] U.K. Köster, J. Meinhard, Crystalization of highly undercooled metallic metals and metallic glasses around the glas transition temperature, Mat. Sci. Eng. A 178, 271-278 (1994).

[29] S. Garus, M. Nabiałek, K. Błoch, J. Garus, The Study of Magnetization in Strong Magnetic Fields for Alloys $\mathrm{Fe}_{60} \mathrm{Co}_{10} \mathrm{~W}_{x} \mathrm{Nb}_{2} \mathrm{Y}_{8} \mathrm{~B}_{20-x}$ $(x=0,1)$, Acta. Phys. Pol. A 126(4), 957-959 (2014).

[30] W.F. Brown, The Effect of Dislocations on Magnetization Near Sturation, Phys. Rev. 60 (2), 139-147 (1941).

[31] W.F. Brown, Theory of the Approach to Magnetic Saturation, Phys. Rev. 58(8), 736-743 (1940).

[32] H. Kronmüller, Micormagnetism and Microstructure of Amorphous Alloys, J. Appl. Phys. 52(3), 1859-1864 (1981).

[33] T. Egami, K. Maeda, V. Vitek, Structural Defects in Amorphous Solids. A computer Simulation study. Philosophical Magazine A41, 883-901 (1980).

[34] D. Goll, Micromagnetism-Microstructure Relations and the Hysteresies Loop. Handbook of Magnetism and Advanced Magnetic Materials, Edited by H. Kronmuller and S. Parkin, John Wiley\&Sons, 2, 1023-1058 (2007).

[35] N. Lenge, H. Kronmüller, Low Temperature Magnetization of Sputtered Amorphous Fe-Ni-B Films. Phys. Status. Solidi A 95, 621-633 (1986).

[36] H. Kronmüller, M. Fahnle, Micromagnetism and the Microstructure of Ferromagnetic Solids. Cambridge University Press, 174-224 (2003).

[37] O. Kohmoto, High-field Magnetization Curves of Amorphous Alloys, J. Appl. Phys. 53(11), 7486-7490 (1982).

[38] T. Holstein, H. Primakoff, Field Dependence of the IntristicDomain Magnetization of a Ferromagnet, Phys. Rev. 58, 1098-1113 (1940).

[39] M. Vazquez, W. Fernengel, H. Kronmuller, Approach to ferromagnetic Saturation in rapidly quenched amorphous alloys, Phys. Stat. Sol. A 115, 547-553 (1989).

[40] F. Carmona, V. Madurga, M. Vazquez, Approach to ferromagnetic saturation in $(\mathrm{Co} 0.95 \mathrm{Fe} 0.05)_{75} \mathrm{Si}_{15} \mathrm{~B}_{10}$ amorphous alloys, J. Magn. Magn. Mater. 62, 68-70 (1986). 